\title{
Porosity Maps - Interactive Exploration and Visual Analysis of Porosity in Carbon Fiber Reinforced Polymers
}

\author{
A. $\operatorname{Reh}^{1}$, B. Plank ${ }^{1}$, J. Kastner ${ }^{1}$, E. Gröller ${ }^{2}$ and C. Heinzl ${ }^{1}$ \\ ${ }^{1}$ University of Applied Sciences Upper Austria, Campus Wels, Austria \\ ${ }^{2}$ Vienna University of Technology, Institute of Computer Graphics and Algorithms, Vienna, Austria
}

\begin{abstract}
In this work a novel method for the characterization of porosity in carbon fiber reinforced polymers (CFRP) is presented. A visualization pipeline for the interactive exploration and visual analysis of CFRP specimens is developed to enhance the evaluation workflow for non-destructive testing (NDT) practitioners based on specified tasks. Besides quantitative porosity determination and the calculation of local pore properties, i.e., volume, surface, dimensions and shape factors, we employ a drill-down approach to explore pores in a CFRP specimen. We introduce Porosity Maps (PM), to allow for a fast porosity evaluation of the specimen. Pores are filtered in two stages. First a region of interest is selected in the porosity maps. Second, pores are filtered with parallel coordinates according to their local properties. Furthermore a histogram-based best-viewpoint widget was implemented to visualize the quality of viewpoints on a sphere. The advantages of our approach are demonstrated using real world CFRP specimens. We are able to show that our visualization-driven approach leads to a better evaluation of CFRP components than existing reference methods.
\end{abstract}

Categories and Subject Descriptors (according to ACM CCS): I.3.6 [Computer Graphics]: Methodology and techniques-Interaction Techniques

\section{Introduction and Motivation}

During the last decades, a clear trend formed in industry of constantly driving industrial research towards new tailored materials as well as cost-effective, function-oriented, highly integrated and light-weight components. The driving forces behind this trend are the high requirements regarding efficiency, environment, safety as well as comfort. Advanced composite materials are very promising allowing to translate the before mentioned high requirements into novel components. Especially carbon fiber reinforced polymers (CFRP) show a great potential due to their increased stiffness and strength-to-weight ratio. As a result of the manufacturing process, these materials tend to have pores inside [MPSH11]. Pores are defined as inclusions of air. Porosity is defined as the volume of pores compared to the volume of the specimen including fibers, resin and pores. Porosity has an impact on the mechanical properties of a specimen such as compressive and interlaminar shear strength as well as the elasticity modulus of the material [BS04]. An important task in quality control is to quantify and study the porosity of samples of such materials. Besides quantitative poros- ity, the distribution and shape factors of pores are important properties for CFRP analysis. For example porosity in a specific layer may be more critical than a homogenous distribution of pores. Based on these requirements, the following tasks for porosity determination and analysis are identified:

- Task 1: The NDT practitioner wants to evaluate a CFRP specimen regarding its quantitative porosity and to compare the results with other reference methods. Therefore a scalar value is sufficient in this case.

- Task 2: For a detailed evaluation of the specimen, a fast overview of the porosity is needed, as the pore distribution influences the local mechanical properties of the component.

- Task 3: To evaluate individual pores, the calculation of local properties is important. In particular the shape factor has a strong influence on mechanical properties and also on the results of reference measurements acquired by ultrasonic testing or active thermography. For example, only big and nodular pores between fiber bundles or thin micro pores between the individual fibers should be analyzed based on the resulting pore classification. 
- Task 4: When evaluating a volume with segmented pores, a good viewpoint is needed. By reason of the analysis definition it should avoid or prefer occlusions and enable the analysis of the majority of pores from the considered viewpoint. Based on the arrangement of the pores, the expressivity of a $3 \mathrm{D}$ view strongly depends on the viewpoint. This is due to overlapping pores in view direction.

In this work, we introduce a visualization pipeline (Figure 1), that is customized for the interactive exploration and visual analysis of CFRP specimens. The workflow is based on X-Ray computed tomography and accomplishes Task 1 Task 4. The main contributions of this paper are:

- Porosity analysis of CFRP is achieved in the precomputation step of the porosity determination. The local pore properties like volume, dimensions and shape factor are calculated automatically and enable a classification of pores.

- Porosity maps allow for fast porosity evaluation of the specimen and for comparison to corresponding ultrasonic and active thermography images. NDT practitioners are able to easily analyze the homogeneity of porosity in the specimen with these maps.

- Interactive exploration of a CFRP specimen is done in a two stages drill-down approach. A region of interest is selected on the porosity maps. Refinement of the selection using parallel coordinates leads to a visualization for an effective porosity analysis.

- Porosity maps are calculated as specific projections along the coordinate axes. These may not be the ideal projection directions. Therefore we propose an optimal projectiondirection search (best viewpoint) which maximizes pore overlap in the result image.

\section{Domain Background}

The methods for porosity determination may be divided into the two groups of destructive and non-destructive testing. Acid digestion and materialography in combination with microscopic analysis belong to the group of destructive testing methods [BS04]. Ultrasonic testing, active thermography and X-Ray computed tomography are non-destructive testing methods [MPSH11].

Voids inside composite materials lead to degradation of strength, fatigue and other deficiency related properties. In high safety applications "zero-tolerance" rules may be required (no voids), or the level of void content has to be below a given level. Less demanding applications can have more tolerant standards, allowing void contents of a few percent $\left[\mathrm{SRvL}^{*} 06\right]$. The currently used state-of-the-art method for non-destructive porosity determination in aeronautics is ultrasonic testing. The porosity level is estimated from ultrasonic velocity and attenuation. Pore distribution, size and shape factor have a strong influence on the ultrasonic attenuation [LLT*08]. The resulting measurement errors have to

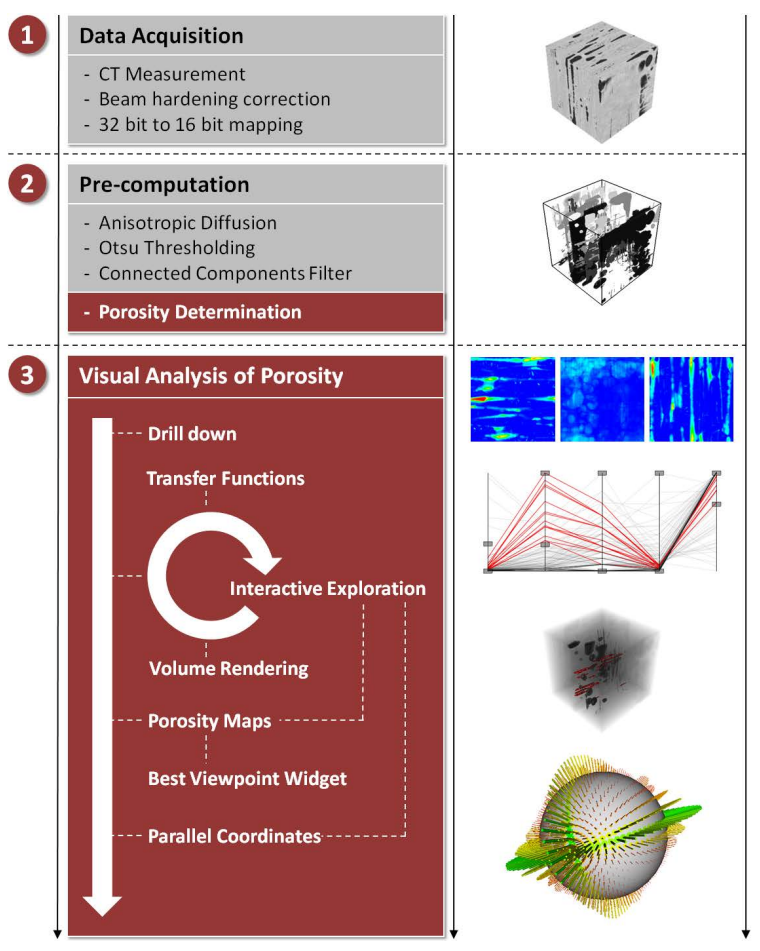

Figure 1: Porosity visualization pipeline for interactive exploration and visual analysis of porosity in CFRP specimens, consisting of three stages: (1) Data Acquisition (2) Pre-computation and (3) Visual Analysis of Porosity.

be considered in quality control. They may lead to a wrong interpretation of porosity and subsequently to a rework or in the worst case to a rejection of possibly useable components. Ultrasonic testing provides standardized means of porosity estimation (Task 1) but an imprecise overview of porosity in the specimen (Task 2). Due to the low resolution the evaluation of specific pores (Task 3 ) is not possible.

Another NDT method for the determination of porosity is active thermography, more specifically pulsed thermography. Generating heat with flashes of light, the propagation of surface temperatures in pulsed thermography depends on the diffusivity of the sample and its geometry. Quantitative porosity is calculated by means of a heat conduction model and the linear relation to the diffusivity [MPSH11]. Compared to ultrasonic testing, active thermography provides an improved porosity estimation (Task 1). It gives a more detailed but still imprecise overview of porosity in the specimen compared to ultrasonic testing (Task 2), but yet no specific evaluation of individual pores (Task 3 ).

Acid digestion is a destructive reference method based on gravimetry. Volume and mass of the specimen, epoxy resin and fiber content have to be measured accurately. Because the specimen is destroyed during the determination, no 
porosity overview (Task 2) and no calculation of local pore properties (Task 3) are available.

In materialography, another destructive method, slices of the specimen are grinded and analyzed with microscopic 2D image processing methods. Porosity is calculated statistically based on a minimal set of 30 slice images. Quantitative porosity can only be determined with reduced accuracy (Task 1) because of grinding artifacts. Due to the estimation being based on a limited number of slices for the whole specimen, a porosity overview (Task 2 ) as well as the calculation of local pore properties (Task 3 ) is complex and timeconsuming.

In recent years 3D X-Ray Computed Tomography (XCT) provides a new possibility for NDT of CFRP components. A specimen is placed on a rotary plate between an $\mathrm{X}$-ray source and a detector. A series of $2 \mathrm{D}$ projection images for many angular positions is taken and the corresponding $3 \mathrm{D}$ volumetric dataset is reconstructed. One of the main problems regarding porosity determination using XCT is the partial volume effect [GPMB95] and the resulting discretization error of the scanned structures. Further the selection of a good viewpoint in the $3 \mathrm{D}$ volume view is important so that the fiber structure is better visible (Task 4).

Another critical problem of all methods is the missing ground truth of quantitative porosity. The accuracy for porosity determination using the reference methods ultrasonic testing and acid digestion [DIN98] is about $\pm 1 \%$.

\section{Related Work}

\subsection{Segmentation}

Segmentation is a common image processing task with a wide range of applications and many approaches are found in the literature. Detailed surveys have been published on GPU-based segmentation by Hadwiger et al. [HLSB04] and on threshold techniques by Sezgin and Sankur [SSO4].

Threshold techniques assign an object membership based on a density threshold, where no neighborhood information is taken into account. Boundary-based methods detect object borders with an edge detection filter and connect the identified edges to form a closed region. A well-established edge detection filter is the Canny edge detector [Can86]. Region-growing methods start from one or multiple regions and grow these regions as long as a homogeneity criterion is valid [AB94] [ZJ07]. The start regions, also called seeds, are manually specified by the user or automatically by an algorithm. A region-based segmentation approach is the watershed transform, where the image is considered as a height field. Beucher applied it to image segmentation [Beu91]. Homogenous regions, also called catchment basins, are constructed by a flooding process. This process leads to oversegmentation. Felkel et al. overcome this problem by placing markers manually [FBW01]. Al-Raoush et al. [ARP10] ap- plied the watershed transform to segment touching or overlapping particles and to calculate the distribution of the local void ratio from $3 \mathrm{D}$ images.

Since region growing methods and the watershed transform are time- and memory-consuming, we used Otsu's thresholding technique [Ots79] for our workflow. In Section 6.1 we show that the results are satisfying compared to the described reference methods.

\subsection{Visualization}

The porosity topic is not limited to material science alone. Seismic engineers use porosity mapping based on geostatistical methods to find oil reservoirs [DL08]. Britz et al. use micro-CT to quantify and visualize cortical bone microstructures $\left[\mathrm{BJL}^{*} 10\right]$.

Due to the availability of several local pore properties, a visualization technique is needed to display multivariate data. Inselberg [Ins09] introduced parallel coordinates, a plot consisting of $n$ parallel axes, depicting an n-dimensional point set. Each local pore property is assigned to a parallel axis. Pore properties are visualized by polylines crossing an axis at the corresponding positions. Parallel coordinates are applied in a wide range of applications. For example, Blaas et al. [BBP08] used parallel coordinates for interactive exploration of large multi-timepoint data sets. Hadwiger et al. [HFRS ${ }^{*} 08$ ] discussed interactive exploration and feature detection of industrial XCT data, including real world applications.

Because of the big number of pores in a CFRP specimen, a drill-down approach is essential for exploring the data. Interactive drill downs using enlarged detail views of the data are employed by Lex et al. [LSP*10] to explore biomolecular data. Yuan et al. [YXG*10] introduce a visual-analytics system including data drill-down for the exploration of seismic data together with satellite-based observational data. For better exploration multiple linked views are essential. SimVis for example uses multiple linked views to explore large sets of data [Dol07].

Regarding best-viewpoint selection Bordoloi and Shen [BS05] presented an entropy-based view selection for volume rendering. Many metrics for image-entropy calculation have been proposed. Typically they are used for image registration of medical datasets [MHV*01] [VWI97]. As proposed by Kohlmann et al. [KBKG07] deformed viewing spheres may be used for navigation in medical datasets. For a better user-oriented adaptability of the metric, we employ a histogram-based approach in our system.

\section{Porosity Visualization Pipeline - Overview}

Figure 1 illustrates the porosity visualization pipeline for quantitative porosity determination and interactive exploration of pores, which is done in three stages. The red background indicates the main contributions of the paper. After 


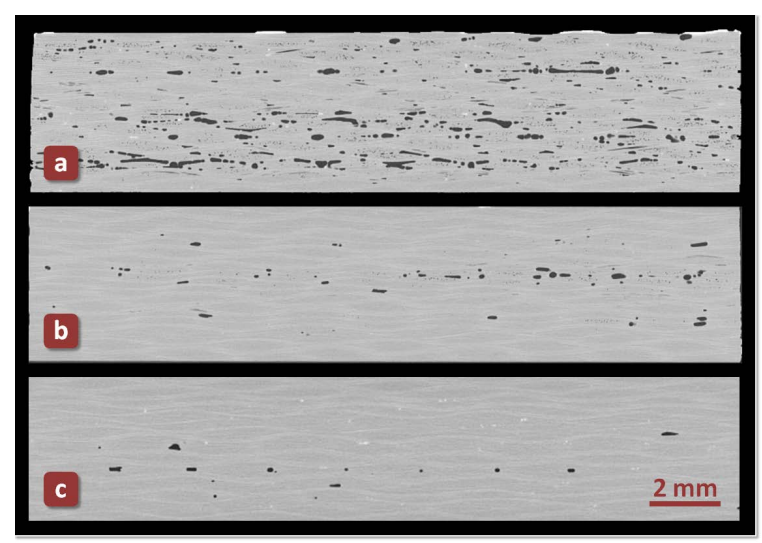

Figure 2: XCT xy slices of specimens PrePreg 1 (a), PrePreg 2 (b), PrePreg 3 (c) contain pores in black. The gray areas indicate the epoxy resin and the fiber bundles.

Data Acquisition (1) (Section 4.1), the calculations required for the interactive exploration and visual analysis of porosity are performed in the Pre-computation (2) stage (Section 4.2). The main stage Visual Analysis of Porosity (3) is described in Section 5. We have integrated the pipeline in our common framework iAnalyse.

\subsection{Data Acquisition}

In this work, samples of preimpregnated fibers (PrePreg) consisting of carbon fibers and 40 weight percent epoxy resin were used to evaluate the quantitative porosity determination. Plates with a porosity between $0 \%$ and $10 \%$ were manufactured by varying the vacuum pressure during the heating phase. For further investigations 6 specimens were cut out of these plates. They are denoted as PrePreg 1-3 with a size of $17 \times 20 \times 4.5 \mathrm{~mm}^{3}$ each and PrePreg 4-6 with a size of $17 \times 20 \times 2 \mathrm{~mm}^{3}$ each. Figure 2 depicts XCT xy slices from PrePreg 1-3 with different levels of porosity. The black holes correspond to pores and the gray areas indicate the epoxy resin and fiber bundles. Single carbon fibers can not be seen, because the voxel size of our measurements is $10 \mu \mathrm{m}$ and the fiber diameter is about $8 \mu \mathrm{m}$. All XCT scans were performed on a GE Phoenix|xray nanotom XCT system with a $180 \mathrm{kV}$ nano focus tube. The reconstruction algorithm of the device included a correction for beam hardening. This avoids over-segmentation in the middle of the specimen due to gray value modifications caused by beam hardening during the measurement.

\subsection{Pre-computation}

Due to ambient noise in the scanned datasets, precomputation is essential for the following segmentation. This step is performed by anisotropic diffusion as described by Perona and Malik [PM90]. Anisotropic diffusion allows smoothing of homogenous regions without blurring edges and leads to improved results for a subsequent analysis of industrial XCT data [HKG07]. We used 5 iterations with a conductance of 1 and a time step of 0.0625 seconds for smoothing the low noise in our datasets by preserving the edges. The segmentation of pores is done with Otsu's thresholding method, which is described in Section 4.2.1. After segmentation a connected-components filter, using 26connectivity, labels objects in the binary image, which is essential for porosity determination (Section 4.2.2).

\subsubsection{Segmentation by Otsu's Thresholding}

Thresholding generates a binary image from a gray level one. Based on a threshold value, all voxels below this threshold are set to 0 and all voxels above are set to 1 . The problem of thresholding is to find the optimal value to classify the voxels into foreground voxels (pores) and background voxels (resin and fibers). Otsu [Ots79] proposed an automatic threshold selection method for bimodal histograms by maximizing the separability of the resulting classes in gray levels. Thereby the histogram is divided into two classes minimizing the intra-class variance and maximizing the inter-class variance.

\subsubsection{Porosity Determination}

To enable interactive exploration and to solve Task 1 and Task 3, we calculate global and local pore properties, which are based on a segmented volume dataset with labeled pores. The following global properties of the specimen are calculated (Task 1):

- Specimen volume $V_{S}$ : The volume $V_{S}$ of the specimen is the sum of all background (epoxy resin and fibers) and pore voxels, but without surrounding air voxels.

- Porosity $P_{s}$ : The specimen porosity $P_{S}$ is calculated as follows:

$$
P_{S}=\frac{V_{P}}{V_{S}}
$$

where $V_{P}$ is the volume of all pores.

Besides global properties, local pore characteristics are calculated for each pore (Task 3) which are then the basis for our parallel-coordinates view:

- Pore volume $V_{P_{i}}$ : The volume $V_{P_{i}}$ of a pore $i$ is the sum of all voxels with the same label, which was assigned by the connected components filter.

- Dimensions $\operatorname{dim}_{x}, \operatorname{dim}_{y}, \operatorname{dim}_{z}$ : The dimensions in $\mathrm{x}, \mathrm{y}$ and $\mathrm{z}$ direction are the projected lengths of a pore along the $\mathrm{x}$, $\mathrm{y}$ and $\mathrm{z}$ axes and are calculated as follows:

$$
\begin{aligned}
& \operatorname{dim}_{x}=\max _{x}-\min _{x}+1, \\
& \operatorname{dim}_{y}=\max _{y}-\min _{y}+1, \\
& \operatorname{dim}_{z}=\max _{z}-\min _{z}+1
\end{aligned}
$$

where $\max _{d}$ is the maximum index and $\min _{d}$ is the minimum index of the pore in the dataset in direction $d$. 


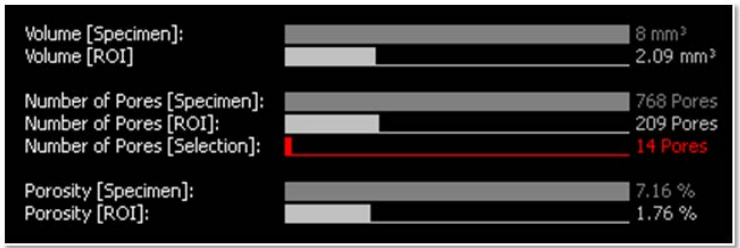

Figure 3: Quantitative porosity info graph showing the global properties volume, number of pores and porosity separately for specimen, region of interest (ROI) and parallelcoordinates selection.
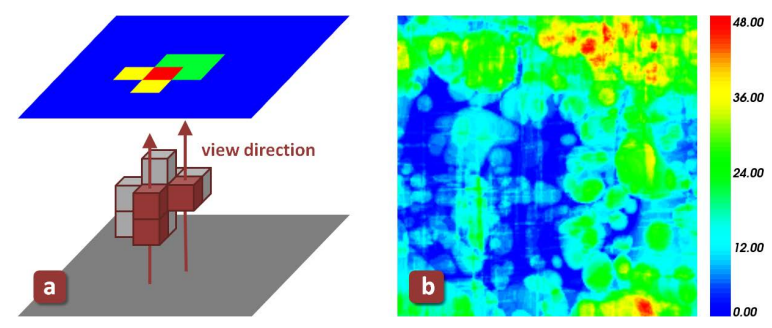

Figure 4: (a) Illustration of porosity map calculation step. (b) View Maximum Color Mapping (VMCM) of a small region in specimen PrePreg 1 in xz view. The porosity map values are encoded as the number of pore voxels.

- Shape factor $F$ : The shape factor $F$ of a pore $i$ is defined as the ratio between the pore surface $S_{P_{i}}$ and the pore volume $V_{P_{i}}$ :

$$
F_{P_{i}}=\frac{S_{P_{i}}}{V_{P_{i}}}
$$

where the calculation of $S_{P_{i}}$ is based on the faces of the surface voxels.

Furthermore porosity maps are calculated for all three orthogonal views. A detailed description of calculation, advantages and color mapping modes of porosity maps is given in Section 5.1.

\section{Visual Analysis of Porosity}

The visual analysis of porosity includes the quantitative and qualitative porosity determination for CFRP specimens, to solve Task 1 - Task 4. The CFRP volume is shown in a 3D view including a best-viewpoint widget and three orthogonal porosity map views, described in Section 5.1. The porosity tab shows an info graph (Figure 3 ) and a parallel-coordinates view for pore filtering (Figure 5 (3)).

\subsection{Porosity Maps}

Porosity maps (PMs) show areas with high and low porosity (Figure 4 (b)). We calculate PMs for the three axis-aligned directions. To calculate an individual pixel of a PM, all pore voxels along a ray in slice direction are summed up. This is shown in Figure 4 (a). Finally the PM values are mapped to colors in one of the following modes:

- Specimen Maximum Color Mapping (SMCM): In this mode the maximum of all three calculated PMs is used for the particular PM color mapping (Figure 5 (1)).

- View Maximum Color Mapping (VMCM): The maximum of each PM is used for its own color mapping (Figure 4 (b)).

Which mode is chosen by the NDT practitioner strongly depends on the component dimensions. If the SMCM mode is selected, colors are consistent in all views. The comparability is ensured, but leads to problems with flat components. Here with SMCM the porosity distribution is difficult or even impossible to identify because of a weak contrast. To ensure comparability to other reference methods of the domain experts we use rainbow color maps.

\subsection{Interactive Exploration and Visualization}

During interactive exploration, the current selection is shown in the 3D volume view. The pores are mapped to color and opacity by a transfer function. Different colors and opacity values for pores inside and outside of the defined region of interest (ROI) as well as selected and unselected pores in the parallel-coordinates view enable a detailed analysis for the user. An info graph and a parallel-coordinates view for interaction complete our visual analysis setup. The axes of the parallel-coordinates view show the five calculated local pore properties, i.e., volume, $\mathrm{x}, \mathrm{y}, \mathrm{z}$ dimensions and shape factor (Figure 5 (3)). The info graph, shown in Figure 3, displays the quantitative porosity to the user, and is helpful when exploring the specimen. It depicts quantitative porosity information for the specimen, for the region of interest and for the parallel-coordinates selection by numerical values as well as by bar charts.

Figure 5 shows the drill-down approach during interactive exploration in three stages. Initially no filtering is applied, so all pores are highlighted (Figure 5 (1)). A detailed porosity overview of the specimen is provided. Based on the porosity maps, the user can interactively specify a ROI in the volume (Figure 5 (a)). In the second stage (Figure 5 (2)) all pores outside the defined ROI are faded out. Simultaneously the parallel-coordinates view is updated, so that only pores inside the ROI are shown. In the third stage (Figure 5 (3)) only selected pores inside the ROI are highlighted. The user can filter pores in the parallel-coordinates view regarding their local properties on the basis of lower and upper bounds (Figure $5(b))$.

\subsection{Best-Viewpoint Calculation}

For the PMs we have so far only considered axis-aligned directions. To increase expressivity of the result images we 

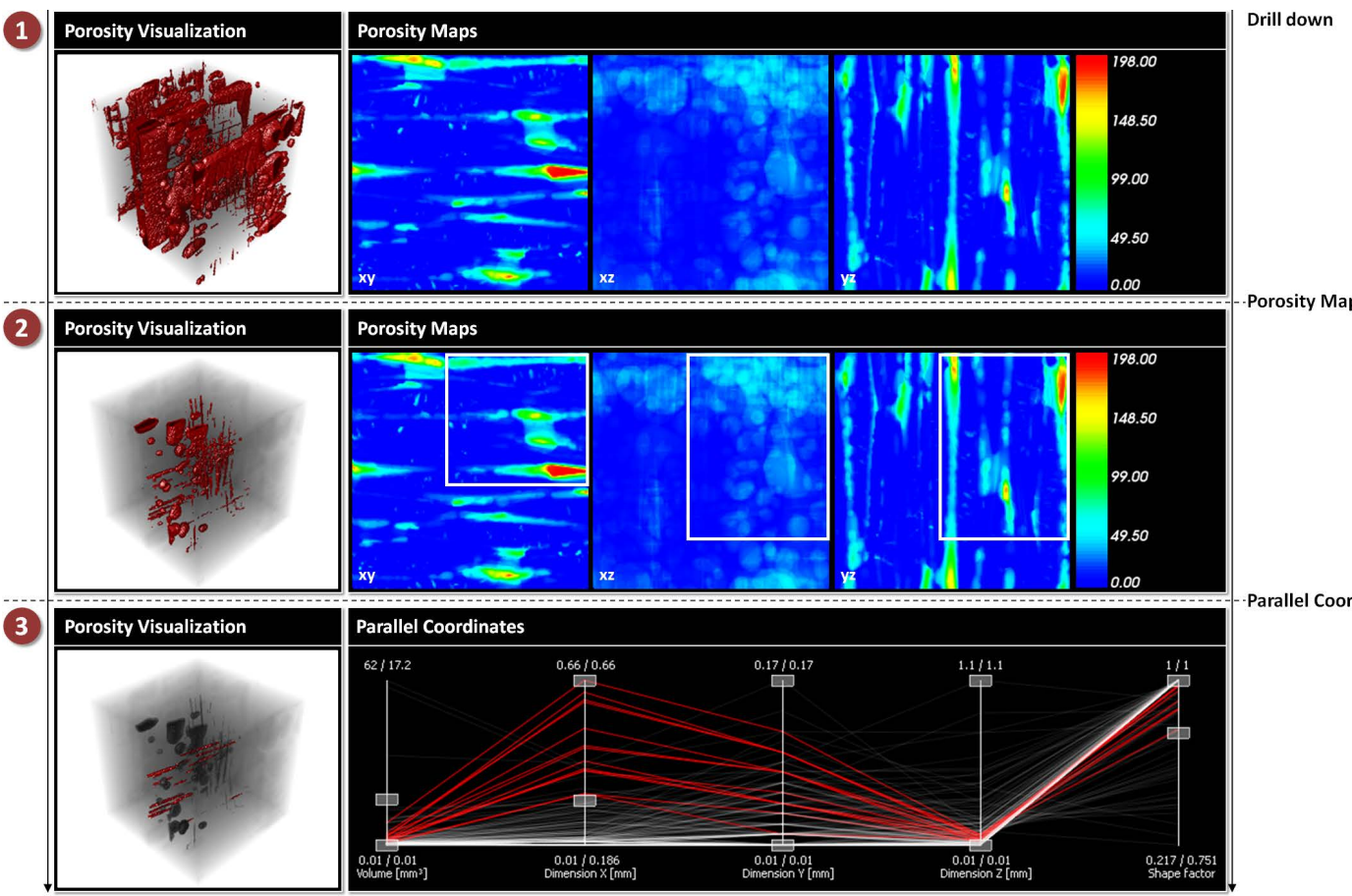

Porosity Maps - ROI Interaction

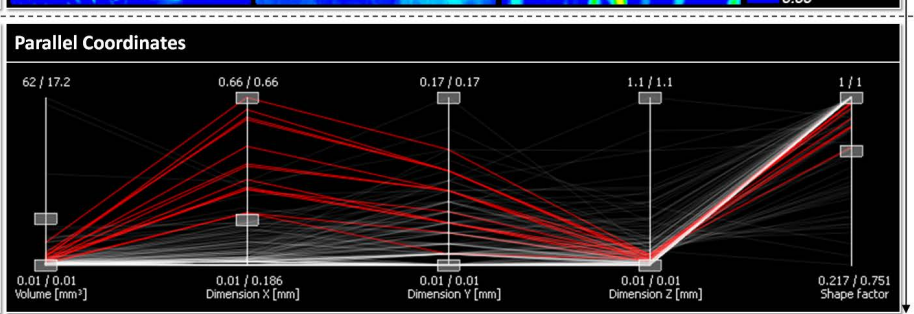

Parallel Coordinates Interaction

Figure 5: Visual analysis of porosity in a sample specimen. The interactive exploration and visualization with a drill-down approach is shown in three stages: (1) Visualization of all pores and fast evaluation of the specimen based on porosity maps, where the values are encoded as the number of pore voxels. (2) Highlighted visualization of pores in a region of interest. (3) Accentuation of specific pores in the region of interest. (a) Porosity maps interaction with a cuboid region of interest. (b) Further filtering of pores based on their local properties using parallel coordinates.

now relax these constraints. We investigate PMs calculated from arbitrary directions (i.e. viewpoints). The goal of the best-viewpoint calculation is to rate the quality of viewpoints (Task 4). In our application domain of CFRP characterization, a good viewpoint maximizes the pore overlap in the result image so that the fiber structure is better visible. Because the quality of a viewpoint greatly depends on the viewed data, the user is able to guide the calculation by parameters.

The main idea is to calculate porosity maps for different viewpoints and to evaluate them. For a fast porosity-map calculation, the opacity of the pores is adjusted in relation to the maximum length of the volume. Overlapping pores cause darker areas in the image. All possible viewpoints are given as a parameterized sphere surrounding the specimen. The direction for the PM calculation is then defined by connecting the viewpoint on the sphere to the sphere center. A discrete set of viewpoints $P_{\alpha, \beta}$ is evaluated by varying the spherical parameters $\alpha, \beta$ in $10^{\circ}$ steps (Figure 6 (a)). For each viewpoint the histogram of the corresponding PM is rated (Figure 6 (b)). We divide the histogram into $n=3$ classes of critical, borderline and not critical pixels using the thresholds $t_{1}$ and $t_{2}$. As critical pixels in black and dark gray are the result of overlapping pores, they make an important contribution to a good viewpoint. Borderline pixels in gray show single pores or pores with less overlapping. Their contribution for a good viewpoint is low. Whereas light gray and white pixels are not critical for a good viewpoint. Based on their contribution to a good viewpoint each class is weighted with the user-defined weights $\omega_{1}, \omega_{2}$ and $\omega_{3}$. The quality value $Q_{\alpha, \beta}$ for a viewpoint $P_{\alpha, \beta}$ is calculated as follows:

$$
Q_{\alpha, \beta}=\sum_{i=1}^{n} \sigma_{i} \omega_{i}
$$

where $\sigma_{i}$ is the sum of pixels in class $i$. Figure 6 (c) shows the best-viewpoint calculation using three viewpoints for a subvolume of PrePreg 1. Therefore the thresholds $t_{1}=50$ and $t_{2}=200$ were used to divide the histograms which are based on 8 bit images. The classes of critical, borderline and not critical pixels were weighted with $\omega_{1}=0.9, \omega_{2}=0.1$ and $\omega_{3}=0.0$.

After calculating $Q_{\alpha, \beta}$ for all viewpoints, the values are visualized on the sphere where we propose two methods. Our first visualization maps quality values $Q_{\alpha, \beta}$ to colors on a sphere (Figure 9 (a)). The second method attaches colored cylindrical sticks to a gray sphere. The length of the sticks 

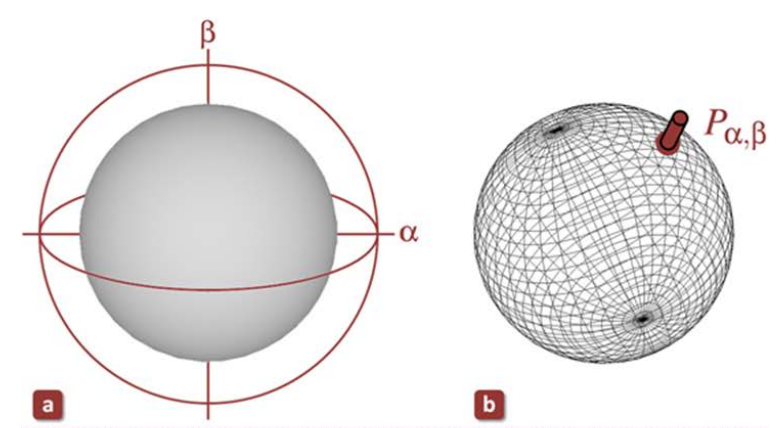

b

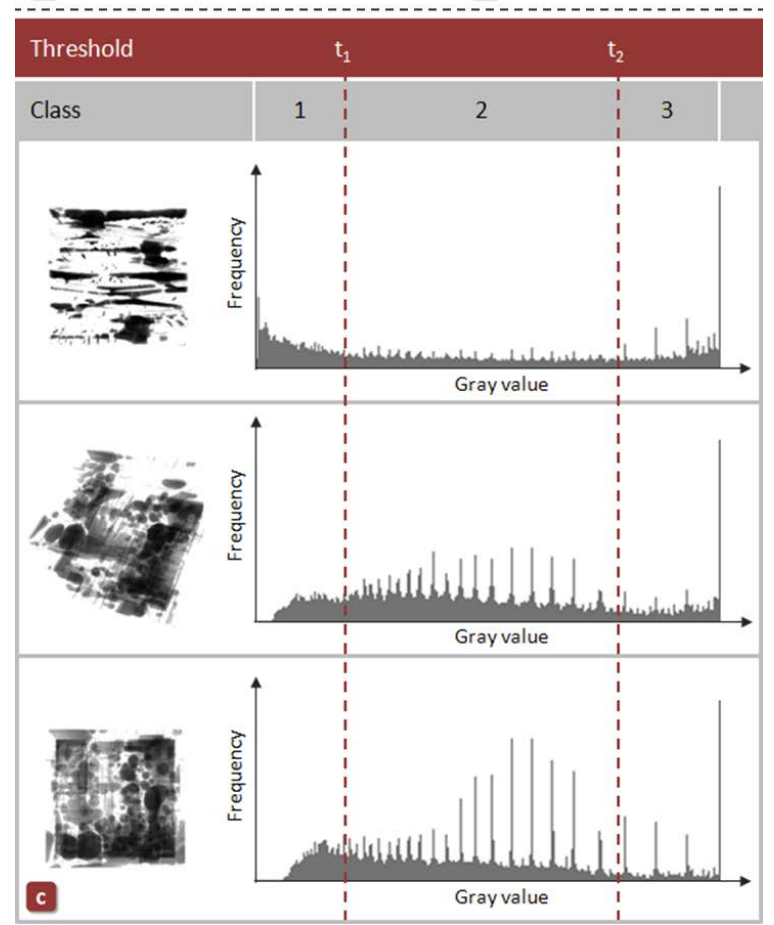

Figure 6: (a) Best-viewpoint calculation by varying the viewpoint in steps of $10^{\circ}$ in $\alpha$ and $\beta$. (b) For each position $P_{\alpha, \beta}$ on the sphere the viewpoint is evaluated. (c) Histogram-based best-viewpoint calculation showing three different viewpoints. Histograms are divided into the three classes of critical (1), borderline (2) and not critical (3) pixels.

also encodes quality values $Q_{\alpha, \beta}$ (Figure 9 (b)). For both methods a color mapping is applied, where views showing the twill-weave pattern of frequently overlapping pores are rated better (in green) than views showing a homogenous image with more visible pores (in red).

\section{Results and Evaluation}

For the practical evaluation of our quantitative porosity determination we compare our results for the three specimens PrePreg 1-3 with the results from the reference methods ul- trasonic testing and acid digestion in Section 6.1. Furthermore the porosity determination is verified using a virtual dataset from a simulated XCT scan (Section 6.2). To show the applicability and the key benefits of our visual analysis approach, we present the exploration of pore classifications on specimen PrePreg 1 as well as the comparison of porosity maps and active thermography images based on the specimens PrePreg 4-6 (Section 6.3). The best-viewpoint widget for a subvolume of specimen PrePreg 1 is discussed in Section 6.4. In Section 6.5 we summarize feedback from CFRP, ultrasonic testing and active thermography experts who assessed the results of our approach. We conclude our evaluation with user feedback about porosity maps in Section 6.6.

\subsection{Quantitative Porosity Determination}

We have calculated the quantitative porosity for the three specimens PrePreg 1-3 (Task 1) using ultrasonic testing, active thermography and XCT. The results are shown in Table 1. Pre-computation times including the anisotropic diffusion filter, Otsu's thresholding, the connected components labeling and the porosity determination strongly depend on the dataset size and the number of pores. For the specimens PrePreg 1-3 the computational time for the porosity deter-

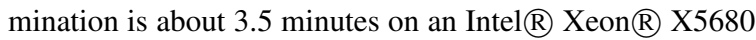
workstation with 48 GB RAM. The comparison to the reference methods ultrasonic testing and acid digestion shows that our XCT-based results are in the range of inaccuracy (about $\pm 1 \%$ ) of ultrasonic testing and acid digestion. The $0.00 \%$ value for porosity in PrePreg 3 measured with acid digestion shows the method's inaccuracy. It may be explained by calculations using inexactly known mass densities of fibers and resin.

\subsection{Segmentation Verification}

For a further quantitative evaluation of the accuracy of the proposed method a small volume was cut out of the PrePreg 3 dataset and reproduced as a virtual specimen with pores given as geometric primitives like cylinders and cuboids. 73 axis-aligned pores were created in the dataset with volumes ranging from $2.8 \times 10^{-5}$ to $3.94 \times 10^{-2} \mathrm{~mm}^{3}$. After a simulated XCT scan generated by the SimCT tool [RHS*10] the porosity result of the simulated dataset with a porosity of $0.198 \%$ was compared to the porosity value of the virtual specimen, where the known porosity (ground truth) is exactly $0.210 \%$. We assume that the deviation of $0.012 \%$ is due to the partial volume effects. The result shows that our segmentation gives satisfying results for the simulated data.

\subsection{Visual Analysis of Porosity}

In this section we present the interactive exploration and visual analysis of a small subvolume of specimen PrePreg 1. Furthermore porosity maps of the specimens PrePreg 4-6 are compared to the corresponding active thermography images. 


\begin{tabular}{lllrrrr}
\hline \hline Dataset & Dataset size [voxel] & $\mathrm{t}$ [min] & No. of pores & Porosity (XCT) & Porosity (US) & Porosity (AD) \\
\hline PrePreg 1 & $1982 \times 458 \times 1673$ & 13.02 & 115165 & $4.57 \%$ & $5.06 \%$ & $3.89 \%$ \\
PrePreg 2 & $2001 \times 452 \times 1686$ & 12.98 & 15772 & $0.81 \%$ & $0.91 \%$ & $0.53 \%$ \\
PrePreg 3 & $1993 \times 441 \times 1701$ & 12.94 & 4167 & $0.39 \%$ & $0.54 \%$ & $0.00 \%$ \\
\hline
\end{tabular}

Table 1: XCT-based porosity results for specimens PrePreg 1-3 compared to the reference methods ultrasonic testing (US) using

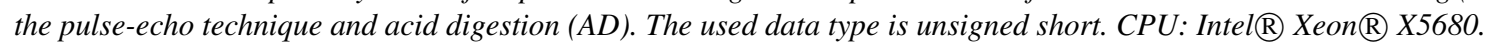
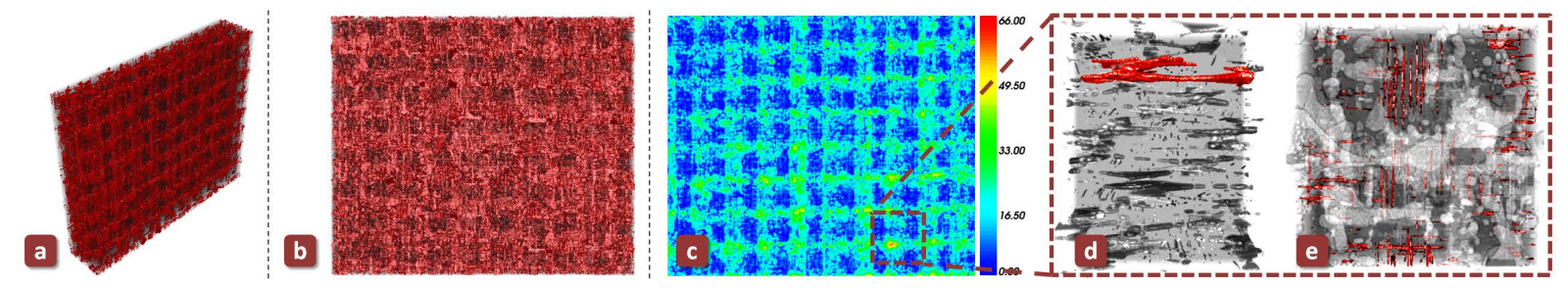

Figure 7: (a) PrePreg 1 specimen after porosity determination in a $3 D$ volume view (b) as well as in a xz view (c) and a xz porosity-map view. The porosity map values are encoded as the number of pore voxels. (d) Porosity evaluation of a region of interest in specimen PrePreg 1. Different pore classifications are shown using parallel-coordinates interaction. The biggest pore in the region is selected and highlighted. (e) Only the long and thin micro pores in the fiber bundles are highlighted.

As an initial task the NDT practitioner has to define a region of interest. Porosity maps show the pore distribution in the whole specimen (Task 2) and hence they are a new way of finding regions with a high porosity. Figure 7 shows specimen PrePreg 1 after our porosity determination in a 3D volume view (a) as well as in a xz view (b). Due to the high number of pores the evaluation of the specimen is a difficult task for the NDT practitioner. The corresponding xz porosity map is shown in Figure 7 (c). It visualizes the pore homogeneity and areas with low (blue) and high (red) porosity. Aligned pores will produce high, hollow peaks in the PM whereas a jittered arrangement of the pores will lead to lower but wider peaks. Based on the porosity map, we cut out a ROI with the highest porosity and show the selection of pore classifications. The use of parallel coordinates allows the user to filter pores with specific pore properties (Task 3). In Figure 7 (d) the biggest pore in the region is highlighted. Figure 7 (e) shows long and thin micro pores in the fiber bundles. For further filtering of macro pores in a specific direction, the dimension bounds can be set in the parallel-coordinates view additionally to the shape factor. Selected pores are highlighted in red. Not-selected pores are given in black, where white and gray illustrate the typical twill weave pattern of the fiber bundles in the component (Figure 7 (d) and (e)). Active thermography images show the temperature distribution that is influenced by the pores. Figure 8 compares such images to porosity maps generated from XCT data. The XCT results show significantly more details. The maximum porosity can be identified more easily as our method uses 3D voxel information based on segmented pores. The structure of the pore network along the twill weave pattern of the fibers is visible due to the better resolution of the XCT measurement $(10 \mu \mathrm{m})$ compared to active thermography (about $150 \mu \mathrm{m}$ ). Further the thermal diffusivity blurs the images.

\subsection{Best-Viewpoint Widget}

For the best-viewpoint visualization the quality of a viewpoint is encoded on a sphere (Task 4). Figure 9 shows our proposed methods for best-viewpoint visualization. In our first approach, viewpoints are color mapped on a sphere (Figure 9 (a). Figure 9 (b) shows a gray sphere with colored cylindrical sticks whose lengths correspond to the calculated quality values. Good viewpoints are shown in green. Red color indicates an unfavorable viewpoint. In our implementation a rotation of the sphere is linked to the corresponding rotation of the specimen.

\subsection{Domain Experts Feedback}

The results generated by the proposed porosity pipeline for XCT data were shown to a domain expert of a company manufacturing aircraft components. In his opinion porosity maps (PMs) are an enrichment providing previously unknown quality and detail in the porosity analysis of CFRP components. The approach allows to escalate and possibly replace common techniques as ultrasonic testing, which is the standard non-destructive testing method in the aeronautic industry. PMs facilitate a fast comparison to the corresponding ultrasonic attenuation images. As a further result, a better exploration of the data allows to draw conclusions about the manufacturing process. Active thermography domain experts mentioned that our visual analysis approach increases their understanding of CFRP components. New findings about the pore distribution can be used to enhance their techniques. 

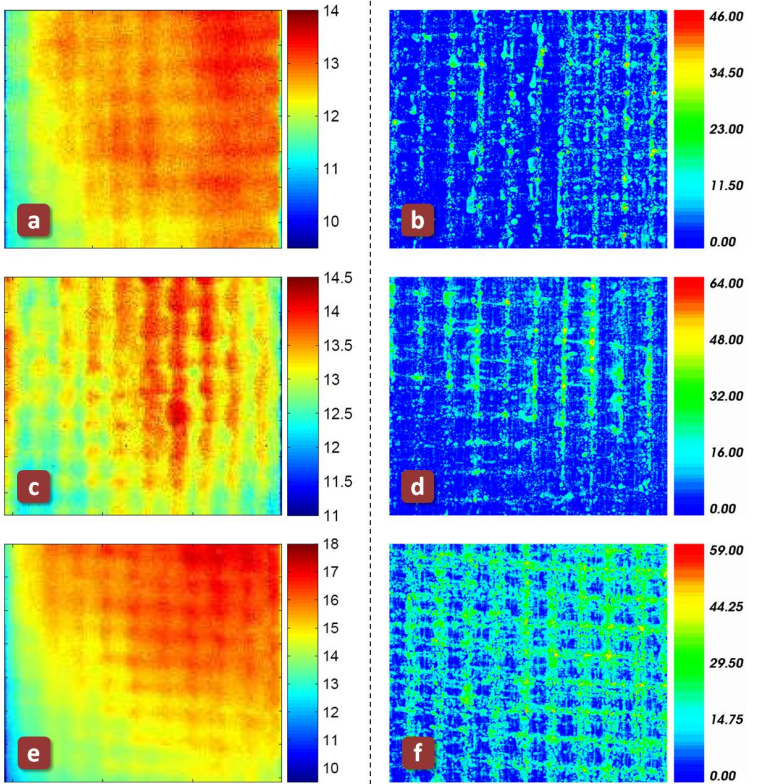

Figure 8: Comparison of active thermography images $(a),(c)$ and $(e)$ to porosity maps $(b),(d)$ and $(f)$ of the specimens PrePreg 4-6. PrePreg 4 shows a porosity of $1.81 \%$ (a) and $(b)$, PrePreg 5 shows a porosity of $3.53 \%(c)$ and $(d)$ and PrePreg 6 shows a porosity of $7.20 \%(e)$ and $(f)$. The porosity map values are encoded as the number of pore voxels. Based on the thermal diffusivity model high values in the active thermography images depict a high porosity, which is encoded as the observation time in seconds.
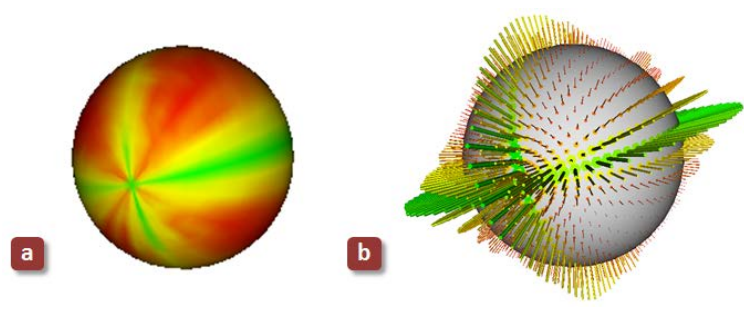

Figure 9: Best-viewpoint widgets with color mapping on a sphere (a) and cylinder sticks (b). The color mapping ranges from green, indicating a good viewpoint, to red visualizing unfavorable viewpoints.

\subsection{User Feedback}

For further evaluation of the porosity-map approach, a questionnaire was filled in by eleven engineering students. The questionnaire consisted of three tasks. In the first task areas with high porosity in images of the specimen PrePreg 1 after porosity determination should be marked. Images from Figure 7 (a-c) showing segmented pores in the 3D volume view, the $3 \mathrm{D} x \mathrm{z}$ view and the corresponding $\mathrm{xz}$ porosity map (without the marked region of interest) were used. The results showed, that the localization of interesting areas with high porosity is easier using porosity maps than with the $3 \mathrm{D}$ views of segmented pores. The second and third task was about the comparison of images from active thermography, ultrasonic testing and porosity maps as well as the identification of related images between the different methods. Three specimens were evaluated. All students preferred porosity maps for finding areas with high porosity, followed by active thermography images. But they were not able to identify correlations between images of the same specimens.

\section{Conclusions and Future Work}

We have presented an approach for the interactive exploration and visual analysis of CFRP specimens. The goal is to enhance the evaluation of such components. Our solution includes porosity determination and the calculation of local pore properties for pore classification and their visualization. The accuracy of the quantitative porosity determination has been shown using real world CFRP parts. The results have been compared to the reference methods ultrasonic testing and acid digestion. Furthermore we compared porosity maps with active thermography images and demonstrated that porosity maps show significantly more details. The introduced pipeline integrates ROI and parallel-coordinates interaction in a two-stage drill-down approach. The bestviewpoint widget allows to see on a sphere the quality varying viewing directions of the corresponding porosity maps. It could be shown, that the combination of porosity maps and interactive exploration is a promising tool for NDT practitioners.

For future work other definitions of shape factors, as used in the field of active thermography, can be included in the porosity determination. As this is the first step for an interactive exploration of CFRP, a rather simple segmentation method was sufficient to show our analysis approach. In the future the segmentation step could be enhanced, to better handle partial volume effects. Moreover local porosity determination for each of the given fiber layers is an interesting topic of research, which has still not been adequately addressed.

\section{Acknowledgements}

This project was supported by the program Regionale Wettbewerbsfähigkeit OÖ 2010-2013, which is financed by the European Regional Development Fund and the Government of Upper Austria. Furthermore this work is partly financed by the K-Project ZPT of the Austrian Research Promotion Agency (FFG). Thanks to the CT group and the active thermography research group of the University of Applied Sciences Upper Austria - Campus Wels as well as FACC AG for measurements, the preparation of CFRP samples and evaluation feedback. 


\section{References}

[AB94] AdAMS R., BISCHOF L.: Seeded Region Growing. IEEE Trans. Pattern Anal. Mach. Intell. 16 (June 1994), 641-647. doi:10.1109/34.295913. 3

[ARP10] Al-Raoush R., Papadopoulos A.: Representative elementary volume analysis of porous media using X-ray computed tomography. Powder Technology 200, 1-2 (2010), $69-77$. doi:10.1016/j.powtec.2010.02.011.3

[BBP08] BlaAs J., Botha C., Post F.: Extensions of Parallel Coordinates for Interactive Exploration of Large MultiTimepoint Data Sets. IEEE Transactions on Visualization and Computer Graphics 14, 6 (November-December 2008), 1436 1451. doi:10.1109/TVCG.2008.131. 3

[Beu91] Beucher S.: The Watershed Transformation Applied To Image Segmentation. In Scanning Microscopy International (1991), pp. 299-314. 3

[BJL*10] BRITZ H., JOKIHAARA J., LEPPÄNEN O., JÄRVINEN T., COOPER D.: 3D visualization and quantification of rat cortical bone porosity using a desktop micro-CT system: a case study in the tibia. Journal of Microscopy 240, 1 (2010), 32-37. doi:10.1111/j.1365-2818.2010.03381.x. 3

[BS04] BiRT E. A., SMith R. A.: A review of NDE methods for porosity measurement in fibre-reinforced polymer composites. Insight - Non-Destructive Testing and Condition Monitoring 46, 11 (2004), 681-686. 1, 2

[BS05] Bordoloi U., SHEN H.-W.: View selection for volume rendering. In Visualization, 2005. VIS 05. IEEE (October 2005), pp. 487-494. doi:10.1109/VISUAL.2005.1532833. 3

[Can86] Canny J.: A computational approach to edge detection. IEEE Trans. Pattern Anal. Mach. Intell. 8 (November 1986), 679-698. doi:10.1109/TPAMI.1986.4767851.3

[DIN98] Aerospace series - Carbon fibre laminates - Determination of the fibre-, resin- and void contents, August 1998. DIN EN 2564:1998-08 (German Industrial Norm). 3

[DL08] Dumitrescu C. C., Lines L.: Seismic attributes used for reservoir simulation: Application to a heavy oil reservoir in Canada. SEG Technical Program Expanded Abstracts 27, 1 (2008), 1471-1475. doi:10.1190/1.3059192. 3

[Dol07] DoleIsCH H.: Simvis: Interactive visual analysis of large and time-dependent $3 \mathrm{~d}$ simulation data. In 2007 Winter Simulation Conference (December 2007), pp. 712 -720. doi: 10.1109/WSC.2007.4419665. 3

[FBW01] FelKel P., Bruckschwaiger M., Wegenkittl R.: Implementation and Complexity of the Watershed-fromMarkers Algorithm Computed as a Minimal Cost Forest. In Computer Graphics Forum (2001), vol. 20, pp. 26-35. 3

[GPMB95] Géraud T., Plurdal L., Maître H., Bloch I.: Estimation of Partial Volume Effect using Spatial Context. Application to Morphometry in Cerebral Imaging. In Nuclear Science Symposium and Medical Imaging Conference Record (October 1995), vol. 3, pp. 1485-1487. 3

[HFRS*08] Hadwiger M., Fritz L., Rezk-Salama C., Höllt T., Geier G., PABel T.: Interactive Volume Exploration for Feature Detection and Quantification in Industrial CT Data. IEEE Transactions on Visualization and Computer Graphics 14 (November 2008), 1507-1514. doi:10.1109/TVCG . 2008.147 .3

[HKG07] Heinzl C., Kastner J., Gröller M. E.: Surface Extraction from Multi-Material Components for Metrology using Dual Energy CT. IEEE Transaction on Visualization and Computer Graphics 13, 6 (November 2007), 1520-1527. doi: $10.1109 /$ TVCG. 2007.70598 .4
[HLSB04] Hadwiger M., LaNger C., ScharSach H., BÜHLER K.: State of the Art Report 2004 on GPU-Based Segmentation. VRVis Research Center (2004). 3

[Ins09] InSELberg A.: Parallel Coordinates: Visual Multidimensional Geometry and Its Applications. Springer-Verlag New York, Inc., Secaucus, NJ, USA, 2009. 3

[KBKG07] Kohlmann P., Bruckner S., Kanitsar A., GRÖLLER M. E.: LiveSync: Deformed Viewing Spheres for Knowledge-Based Navigation. IEEE Transactions on Visualization and Computer Graphics 13 (October 2007), 1544-1551. doi:10.1109/TVCG.2007.70576. 3

[LLT*08] Lin L., Luo M., Tian H. T., Li X. M., Guo G. P.: Experimental investigation on porosity of carbon fiber-reinforced composite using ultrasonic attenuation coefficient. In $17^{\text {th }}$ World Conference on Nondestructive Testing, Shanghai (2008). 2

[LSP*10] Lex A., Streit M., Partl C., Kashofer K., Schmalstieg D.: Comparative Analysis of Multidimensional, Quantitative Data. IEEE Transactions on Visualization and Computer Graphics 16, 6 (November-December 2010), 1027-1035. doi:10.1109/TVCG.2010.138.3

[MHV*01] Mattes D., Haynor D. R., Vesselle H., LEWELlen T. K., EubANK W.: Nonrigid Multimodality Image Registration. Proceedings of Medical Imaging 4322 (2001), 1609-1620. 3

[MPSh11] Mayr G., Plank B., Sekelja J., Hendorfer G.: Active thermography as a quantitative method for nondestructive evaluation of porous carbon fiber reinforced polymers. NDT \& E International 44, 7 (2011), 537-543. 1, 2

[Ots79] OTSU N.: A threshold selection method from gray level histograms. IEEE Trans. Systems, Man and Cybernetics 9 (March 1979), 62-66. 3, 4

[PM90] Perona P., Malik J.: Scale-Space and Edge Detection Using Anisotropic Diffusion. IEEE Trans. Pattern Anal. Mach. Intell. 12 (July 1990), 629-639. doi:10.1109/34.56205. 4

[RHS*10] Reiter M., Heinzl C., Salaberger D., Weiss D., KASTNER J.: Study on parameter variation of an industrial computed tomography simulation tool concerning dimensional measurement deviations. In $10^{\text {th }}$ Conference on Non-Destructive Testing (Moscow, June 2010), p. pp. 8.7

[SRvL*06] SChell J. S. U., RengGli M., VAN Lenthe G. H., MÜller R., ERmanni P.: Micro-computed Tomography Determination of Glass Fibre Reinforced Polymer mesostructure. Composites Science and Technology 66, 13 (2006), 2016-2022. doi:10.1016/j.compscitech.2006.01. 003.2

[SS04] SEZGIN M., SANKUR B.: Survey over image thresholding techniques and quantitative performance evaluation. J. Electronic Imaging 13, 1 (2004), 146-168. 3

[VWI97] Viola P., Wells III W. M.: Alignment by Maximization of Mutual Information. International Journal of Computer Vision 24, 2 (1997), 16-23. 3

[YXG*10] Yuan X., Xiao H., Guo H., Guo P., Kendall W., HuAng J., Zhang Y.: Scalable Multi-variate Analytics of Seismic and Satellite-based Observational Data. IEEE Transactions on Visualization and Computer Graphics 16, 6 (NovemberDecember 2010), 1413 -1420. doi:10.1109/TVCG.2010. 192.3

[ZJ07] Zhigeng P., JiAnfeng L.: A Bayes-Based RegionGrowing Algorithm for Medical Image Segmentation. Computing in Science and Engineering 9 (July 2007), 32-38. doi: $10.1109 / \mathrm{MCSE} .2007 .67 .3$ 